\title{
Thinking Globally, Acting Locally
}

\section{ROCKEFELLER UNIVERSITY'S ENTERPRISING CTSA WORK}

BARRY S. COLLER, MD

T he goals of the Rockefeller Clinical and Translational Science Award (CTSA) include both transforming clinical and translational research at Rockefeller University and contributing to CTSA-wide efforts to reengineer the clinical research enterprise at the national and international levels. In this article, I will highlight some of the national initiatives Rockefeller University in New York, New York, is pursuing.

\section{Standards and Certification for Clinical Research Nursing}

The clinical research nurse occupies a central role in the clinical research process, serving both as the clinical caregiver and as a member of the research team.

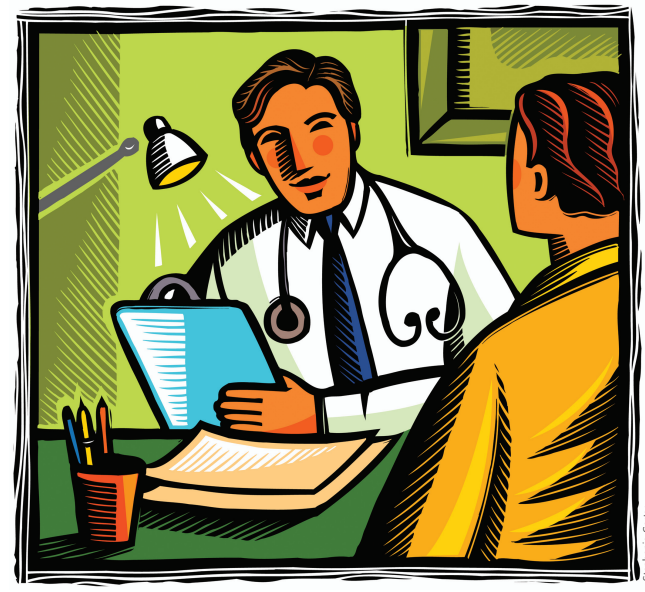

Rockefeller University is developing methods to improve datagathering in clinical research by exploring the types of data collected and collection methods.
Gallin, MD, David Henderson, MD, and Laura Lee, RN-began to address this need by adding questions to their general hospital patient satisfaction survey that asked about the participant's research experience. Rockefeller University-led by Cindy Seidman, director of regulatory affairs; Rhonda G. Kost, MD, clinical research officer; Barbara O'Sullivan, $\mathrm{MD}$, hospitalist and CEO; and myselfjoined this effort and deployed a similar instrument at Rockefeller. The ability to benchmark against another research-intensive clinical facility was particularly valuable, and important data were obtained that resulted in new educational efforts and policies. There were, however, several major limitations The former role requires general nursing knowledge and skills, including patient advocacy, whereas the latter requires advanced level knowledge and skills related to the conduct of clinical research.

Several years ago, Kelly McClary, director of nursing and patient care services at Rockefeller, began to meet with a group of nursing colleagues at other academic clinical research institutions and the Clinical Center at the National Institutes of Health (NIH) to discuss the development of clinical research nursing as a specialty practice. McClary left Rockefeller for Switzerland in 2006 and her successor, Melissa Offenhartz, has carried on this important leadership role with the working group.

The goals of the working group are to develop a curriculum and an accompanying textbook, suggest standards of training, and advocate for certification by the American Nursing Association of the nursing specialty practice of clinical research nursing. Currently, the working group contains representatives from 9 CTSAs and the NIH Clinical Center. Ultimately, the working group plans to disseminate all of its materials publicly, with a particular focus on other CTSA institutions. For additional information, contact Offenhartz at moffenhartz@rockefeller.edu.

\section{Participant Perception Questionnaire}

One of the major obstacles to improving the clinical research enterprise is a lack of information about how clinical research is perceived by the research participants. For example, knowing that an informed consent form was properly signed (a "process" quality control indicator) does not provide information about whether a participant understood that he or she could leave the study at any time (an "outcome" indicator). More than 5 years ago, the leadership of the NIH Clinical Center-including John of this approach - namely, the limited number of sites and the lack of a formal process to develop and validate the questions.

To address these limitations, Dr. Kost has led a consortium of CTSA and GCRC sites to develop and deploy a comprehensive survey containing validated questions about the research participant experience. From its beginning, this project has been a public-private partnership with the NRC Picker Corporation, which produces, validates, and analyzes patient satisfaction surveys. Fourteen centers (including CTSAs and GCRCs) and the NIH Clinical Center participated in the early project development, and 8 centers are actively involved in the first phase of the project.

The question development process is now well underway; the process includes 22 focus groups of research participants and academic leaders in the field of the protection of human subjects at 8 sites around the country. After the draft survey instrument is produced, it will be deployed on an experimental basis in a larger cohort of participating institutions. Individual sites fielding the survey will receive information on their center's performance on a confidential basis, and centers will have the ability to privately compare their results to the aggregate score of the group. This will allow centers to benchmark against peer institutions. Going forward, centers may analyze trends over time to measure the effectiveness of process and educational interventions and to document their performance to agencies accrediting their research protection programs. Ultimately, our goal is for NRC Picker to offer the survey and a detailed analysis of the results to all interested institutions at a competitive cost so as to maximize its utility for the entire research enterprise.

\section{Rockefeller University Phenotyping Initiative}

Clinical and translational research depends on careful clinical 
observations of patients. The organization, storage, and retrieval of these observations, however, has been a major challenge. Medical records traditionally have been used for this purpose, but the information is often inadequate for research purposes. With the explosion of genetic knowledge, it is now clear that patients with the same genetic abnormality may have very different phenotypes. For example, 2 patients with sickle cell disease may have dramatically different clinical courses, with one experiencing frequent severe pain episodes and an early death and the other having very few pain episodes and a near-normal life span. Thus, other genetic, environmental, and perhaps stochastic phenomena affect the phenotype.

Historically, some of the most successful clinical and translational projects have achieved their value by virtue of their recording, storing, and retrieving phenotypic information. The Framingham study, which defined many important cardiovascular risk factors, has not only dramatically affected the clinical care of patients but also provided the impetus for basic science programs to develop drugs to lower blood cholesterol. Recent advances in genome-wide association studies also highlight the importance of the ability to study large numbers of patients from different ethnic and racial groups around the world, as some patterns of association will only emerge from such large data sets. Finally, the lack of readily accessible standardized methods to record clinical observations also serves as a major impediment to junior investigators entering the field of clinical and translational research.

Clinical and translational research depends on careful clinical observations of patients. The organization, storage, and retrieval of these observations, however, has been a major challenge.

The Rockefeller University Phenotyping Initiative is designed to address many of these issues. The centerpiece is a formal ontology describing all of the phenotypic elements considered relevant for assessing a particular disease or biologic process and the relationships that exist between or among the elements. Alongside the ontology is a clinical questionnaire designed to elicit information from a patient or research participant about the relevant clinical manifestations. This questionnaire is then converted into an electronic Web-based or freestanding application (Phenotype Recording and Analysis Tool, or PRAT) that is used to collect the data from the patient or participant. To standardize the language, all medical terms are mapped to the terms defined in the Unified Medial Language System (UMLS).

The initial goal of the project is to make Phenotype Recording Instruments freely available to investigators around the world, thus encouraging their use by multiple investigators. The ontologies will be submitted to the National Center for Biomedical Ontologies (NCBO). We will make the questionnaires and PRATs available as well, either through $\mathrm{NCBO}$ or directly to requesting investigators. Our ultimate goal is to have investigators upload their deidentified phenotypic, genotypic, proteomic, imaging, and pathologic data into a common data repository, permitting the aggregation of data from multiple sites. This would dramatically enhance the statistical power of the studies. Moreover, the database would continue to grow over time and prove ever more valuable. An array of biostatistical tools can be applied to these data sets depending on the goals of the investigator.

As a first step in realizing this vision, I, because of my expertise in the field of clinical hemostasis and thrombosis, worked with the Bioinformatics Group at Rockefeller-consisting of Edward Barbour, MS, Nickolay Khazanov, MS, and Natasha Levenkova, MS, as well as a clinical scholar, Andreas Mauer, MD—to develop a Phenotype Reporting Instrument for obtaining information on a person's bleeding history. Daniel L. Rubin, MD, research scientist and clinical assistant professor of radiology, and Mark Musen, $\mathrm{MD}, \mathrm{PhD}$, professor of medicine, both of Stanford University in Stanford, California, who are researchers at NCBO and leaders in the field of medical ontologies, have provided valuable advice on the conceptualization of the project and the design of the bleeding history ontology, questionnaire, and PRAT.

Currently, we plan to use the PRAT to obtain bleeding history information from normal individuals of different ages, sexes, and racial and ethnic backgrounds. We also will make these data available to other investigators. There are many ways we plan to use the information we obtain with the aid of the Phenotype Reporting Instrument. These include:

1 Define the normal range and variability of bleeding symptoms by age, sex, and racial and ethnic background;

2 Identify the patterns and severity of symptoms associated with different hemostatic disorders;

3 Define the optimal criteria to separate the normal population from the population of patients with one or another bleeding disorder;

4 Correlate individual symptoms or groups of symptoms with biochemical or genetic data to identify the dominant relationship;

5 Identify individuals whose phenotypes fall far outside what is expected from the dominant relationship for further analysis of genetic or environmental influences that may affect the phenotypic expression;

6 Identify the fewest number of questions that can be asked to exclude the diagnosis of a significant hemostatic defect with reasonable statistical certainty, minimizing the amount of time a clinician requires to make this assessment; and

7 Define criteria to decide whether to initiate an extended (and expensive) laboratory evaluation for a bleeding disorder, thus enhancing the cost-effective use of scarce medical resources.

We plan to share the data we obtain from this pilot project with the CTSA consortium on an ongoing basis. If other CTSA investigators wish to use our system, we will provide support, and if other CTSA investigators wish to create an ontology, questionnaire, and PRAT for the disease that they are studying, we can offer advice. CTS 